Notre Dame Journal of Formal Logic

Volume XXI, Number 1, January 1980

NDJFAM

\title{
THE LOGIC OF RELATIVE MODALITY AND THE PARADOXES OF DEONTIC LOGIC
}

\author{
JOHN BRYANT
}

Summary Arguments of Hamlyn indicate that a radical revision of modal logic should be undertaken. Suggestions for such a revision are developed briefly here; and using these suggestions, it is found not only that deontic logic reduces to modal logic, but also that three troublesome paradoxes of the former-Chisholm's Paradox, Hintikka's Paradox, and the Paradox of the Good Samaritan-can be resolved without difficulty. In the final section, a number of other relevant problems are discussed, and solutions for them are proposed. These include Chisholm's problem of doing otherwise, the problem of future contingency and necessity, the problem of deriving "ought" from "is", and the problem of nested modal expressions.

A Relative Modality David Hamlyn [6] has argued convincingly that the only reasonable way to interpret the assertion that a statement is "necessary" is to say that the statement is necessary relative to some other statement. This thesis, however, would seem to entail a radical revision of modal logic, for it would seem to require that every modal statement be asserted with an explicit reference to the statement relative to which the original statement is asserted. That is, rather than writing the ordinary modal expressions " $p$ is necessary" and " $p$ is possible", it would seem to be required that we instead write something like " $p$ is necessary relative to $q$ " and " $p$ is possible relative to $q$ ". If we do this, however, and if we consider the necessity involved to be logical necessity (as opposed to physical necessity, which we shall discuss shortly), the most natural interpretation of relative modal expressions would seem to involve implication, as in Table 1.

Table 1

$\begin{array}{ccc}\text { Old Form } & \text { New Form } & \text { Interpretation } \\ & p N q & q \rightarrow p \\ -\diamond p & p-P q & q \rightarrow-p \\ \diamond p & p P q & -(q \rightarrow-p) \\ -\square p & p-N q & -(q \rightarrow p)\end{array}$

Received February 20, 1976 
where the last two interpretations are of course derived by taking the appropriate denials of the others. ${ }^{1,6}$

Now one potential objection to the interpretations of Table 1 is that, given $p P q$, one might wonder whether it is not still possible that $p-P q$, for certainly in ordinary language, when we say something is possible, we do not mean to deny that at some later date it might be discovered to be impossible; but by using Table 1 , if we say that $p$ is possible, then this is tantamount to denying that $p$ is impossible. Now the best resolution to this problem, so it would seem, is that when we say that $p P q$, and also argue that it is "possible" that $p-P q$, the term in quotation marks is really a different sort of possibility from that asserted in $p P q$. The quotationmarked possible is a metapossibility which takes into account such things as that we may have made a mistake in arriving at $p P q$. A metapossibility, however, does not participate in the logical system in which $p P q$ is found, and thus there exists no contradiction.

But even if we adopt the conventions of Table 1 for logical necessity, it remains to be answered how we should handle statements of physical necessity such as

1. Water is (physically) necessary for a man to survive.

Now as is well-known, David Hume [7] (see also [12], pp. 58-9) argued against the practice of saying that effects are "necessary" results of their causes on the basis that empirical connections are matter-of-fact ones, and thus that there is no contradiction or other difficulty in supposing a given cause to have some effect other than the one which it allegedly makes "necessary." This argument may of course be extended against the notion of physical necessity, in the sense that it seems more economical to speak merely of empirical facts and apparently invariable regularities rather than "necessary links" between states of affairs. From this viewpoint, therefore, it would seem that (1) should be rewritten as

2. A man who survives has water.

A further reason for avoiding statements of physical necessity is that such a practice avoids the otherwise apparent need to equate part or all of the "necessary/possible/impossible" trichotomy with the "certain/possible/impossible" trichotomy, when the former would seem to apply most appropriately to analytic statements and the latter to synthetic ones. ${ }^{2}$

Yet a third argument for rejecting "physical necessity" as a proper subject of modal logic can be based upon an argument which $\mathrm{I}$ have given in greater detail in [2]. The two fundamental points of this argument are as follows: (1) All statements appear to have three (roughly) equivalent forms: hypothetical, implicative, and categorical. The hypothetical statement is of the form "If $A$ then $B$ ", the implicative statement is of the form " $A$ implies $B$ ", and the categorical statement is one having neither a hypothetical nor an implicative form. (For example, "All crows are black" is the categorical form of a statement whose hypothetical and implicative forms are "For all $x$, if $x$ is a crow then $x$ is black", and "For all $x$, that 
$x$ is a crow implies that $x$ is black.") (2) If a statement is considered analytic, its implicative form is most appropriate; if synthetic, then its categorical form is most appropriate. ${ }^{3}$ The hypothetical form is "neutral". (For example, if part of the definition of "crow" is "being black", then "All crows are black" is analytic, and thus it is appropriate to render it in the implicative rather than the categorical form, since the statement is true by virtue of (a logical) implication. But if the statement is instead considered an empirical report (i.e., is synthetic), then the categorical form is evidently more appropriate.)

Now the above theory can be employed to argue against the concept of physical necessity, as follows: Statements of physical necessity appear to be mere deviant forms of categorical statements, as can be seen by comparing statement 1 with the categorical 2 . In addition, statement 1 contrasts with statements of logical necessity (which we take as relative necessity, of course) such as

3. 7 is (logically) necessarily less than 9 (relative to the axioms and definitions of mathematics),

which is most reasonably written as an implicative (rather than a categorical) form:

4. The axioms and definitions of mathematics imply that 7 is less than 9 .

In short, statements of physical necessity are best considered as mere categorical statements (in which the reference to "necessity" does not appear), while statements of logical necessity are best handled as implicative statements, the (relative) necessity being translated into an implication. Accordingly, physical necessity may be dispensed with, leaving logical necessity as the only object to which modal logic ought to be applied.

B Deontic logic It has been noted by several writers on the topic that deontic logic, i.e., the logic which deals with the logical (as opposed to ethical) relations of such ideas as obligation, permissibility, and forbiddenness, is analogous in many respects to modal logic. For example, the following statements of deontic logic

1. What is obligatory is permissible.

2. What is not permissible is what one is obliged not to do, and vice versa.

3. What we are committed to by something obligatory is itself obligatory.

bear an obvious relation to the following statements of modal logic:

4. What is necessary is possible.

5. What is impossible is necessarily false and vice versa.

6 . What is necessitated by something necessary is itself necessary. 
Because of such relations it has been suggested that deontic logic may be a "branch" of modal logic; and it is my intention in the following section to argue that this is in fact the case, under the following assumptions: (1) Modal logic is analysable in the manner suggested in Section A; (2) " $x$ is obligatory" is construed as " $x$ is necessary with respect to avoiding penalty" ( $(x \mathrm{Na}),(3)$ " $x$ is forbidden" is construed as " $-x$ is necessary (or $x$ is not possible) with respect to avoiding penalty" ( $-x \mathrm{Na}$ or $x-P a$ ), and (4) " $x$ is permissible" is construed as " $x$ is possible ( $-x$ is not necessary) with respect to avoiding penalty" ( $x \mathrm{~Pa}$ or $-x-\mathrm{Na})$. In addition, we shall assume that "penalty" is here construed as "punishment or possible punishment" or something of the sort, in order to overcome the objections raised by some philosophers that, since a wrongdoer often escapes punishment, we cannot say that any actions are strictly necessary for avoiding punishment. In other words, we are saying that the existence of the possibility of punishment is also "punishment", in some appropriate sense of "possibility" and "punishment". (Note that we must say "in some appropriate sense of "possibility"' since, even though one may not have committed an act subject to punishment, it is still possible in some sense for one to be punished, but this is not the same sense in which we say that it is "possible" that one will be punished given that he has committed a wrongful act.)

The reason some philosophers have felt that deontic logic does not correspond in all respects with modal logic is that although

7. " $x$ is necessary" entails " $x$ is actually the case" entails " $x$ is possible",

it does not appear true that

8. " $x$ is obligatory" entails " $x$ is actually done" entails " $x$ is permissible".

However if we reinterpret 7 and 8 as

$7.1(x N a) \rightarrow(x$ is true with respect to $a) \rightarrow(x P a)$

$8.1(x N a) \rightarrow(x$ is actually done with respect to $a) \rightarrow(x P a)$

then we see that the correspondence is essentially exact. It may, incidentally, be instructive for the reader to write out the proofs for 7.1 and 8.1, and also the proofs for the proper interpretations of 1-3.

C Three paradoxes Three well-known problems which have plagued deontic logic are Hintikka's paradox, Chisholm's paradox, and the paradox of the Good Samaritan (see [10]). The present section explains how these paradoxes may be resolved using the theory we have so far developed.

Hintikka's paradox may be explained as follows: It would seem reasonable for one to assume that the following statement is true:

1. If $x$ is a wrong act, and if it is impossible to do $y$ without doing $x$, then it is wrong to do $y$. 
Given, however, that $y$ is impossible, the following statement is true independently of statement 1 :

2. It is impossible to do $y$ without also doing $x$.

Of course the reason statement 2 is true is that if doing $y$ is impossible, then clearly so is both doing $y$ and doing $x$; and this therefore means that 2 is true when $y$ is impossible, irrespective of whether $x$ is wrong or not. This, however, leads to the conclusion that, since statement 1 is true both when $y$ is possible and when $y$ is impossible, then 1 entails that it is wrong to do what is impossible. This, however, is absurd, and in fact is worse than absurd, since if we assume that

3 . If $x$ is wrong, then attempting to do $x$ is wrong,

then if we should attempt (however innocently) an impossible act such as the proof of a theorem which happens in fact to be false, then it would follow that we would have committed wrong.

Now in terms of our present theory, Hintikka's paradox may be formulated (using statement 1 ) in either one of the following two ways:

4. $(-x N a) \cdot(y-P a) \cdot(y \cdot-x-P a) \rightarrow-y N a$

5. $(-x N a) \cdot(y \cdot-x-P a) \rightarrow-y N a$

where 4 includes the assumption that $y$ is impossible while 5 represents the more general case in which no assumption about $y$ 's possibility is made. Both statements are true, and of course both have the paradoxical conclusion that doing $y$ is wrong.

$$
5.1(a \rightarrow(-x \cdot y \cdot-y)) \rightarrow(a \rightarrow-y) .
$$

Now the resolution of the paradox hinges on recognizing the tacit assumption exhibited in both 4 and 5, that both the wrongness of $x$-which we have interpreted as the necessity of $-x$ with respect to $a$-and the impossibility of $y$ have been taken relative to the same proposition, viz., a, when it is evident on reflection that the wrongness of $x$, i.e., the moral necessity of $-x$, is necessary relative to something different than the (synthetic or analytic) necessity of $-y$. That this in fact resolves the paradox may be seen by observing that a reformulation of 4 and 5 in light of the foregoing as

6. $(-x N m) \cdot(y-P a) \cdot(y \cdot-x-P a) \rightarrow-y N a$

and

7. $(-x N m) \cdot(y \cdot-x-P a) \rightarrow-y N a$

are both false as long as it is not the case that either $m \rightarrow a$ or $a \rightarrow m$.

Chisholm's paradox and the paradox of the Good Samaritan devolve from much the same confusion as Hintikka's paradox. The latter paradox may be explained as follows: Suppose I am helping someone who is being attacked. In this case, my helping the person who is being attacked entails that a person is being attacked, and hence that a wrong is being done; thus my right action entails a wrong action, and is therefore objectionable by the 
principle that whatever entails the doing of something wrong is itself wrong. The fallacy of this reasoning may be made plain by letting $a$ and $h$ represent the situations of the attack and my helping, respectively, and symbolizing what is presumed to be the argument as follows:

8. $(-a N s) .(h \rightarrow a) \rightarrow(-h N s)$

or

$$
8.1(s \rightarrow-a \rightarrow-h) \rightarrow(s \rightarrow-h)
$$

which is a valid entailment. It seems clear from 8.1, however, that $s$, which is the proposition that the necessity of $-a$ is assumed relative to, is not a moral code, but a state of affairs of the world, and that while 8 is valid, the fact is that $s$ is false, i.e., the state of affairs in which the attack does not take place does not hold. In other words, what 8 says is that if there is no attack, then I offer no help; and this is not a paradox. This, however, means that 8 does not symbolize the paradox, as had been presumed. But what does? The answer is found in realizing that my helping is relative to both my being moral $(m)$ and the existence of the attack $(a)$; thus the argument is properly symbolized as

$$
\text { 9. }(m) \cdot(a) \cdot(h N a \cdot m) \rightarrow h
$$

which is valid by modus ponens.

Chisholm's paradox may be explained as follows: It seems reasonable to assume that

10. If we ought to do $x$, and if it ought to be that we do $y$ given that we do $x$, then we ought to do $y$.

Chisholm's paradox consists essentially in asking the question "Ought we do $y$ ?', but asking it divorced from any recognition that the question can only be answered for certain if we know we have done $x$. (In the case where we do not know whether we have done $x$, the question is unanswerable.) That is, although Chisholm uses 10 as a premise, he interprets "Ought we do $y$ ?" as an "absolute ought", i.e., as a question of ought which is not relative to anything save 10. It is evident, therefore, why Chisholm's question has been thought paradoxical.

From the above considerations it would seem reasonable to inquire as to whether the relation of entailment itself should be considered to hold or not relative to some set of conditions, i.e., whether every entailment $A \rightarrow B$ must be assumed to be of the form

$$
\text { 11. } K \rightarrow(A \rightarrow B)
$$

where $K$ is interpreted as the set of conditions required for $A \rightarrow B$ to be true. This, however, evidently engenders an infinite regress, since 11 is also an entailment, and thus that there must be some $K^{\prime}$ relative to which 11 holds, i.e.,

$$
\text { 12. } K^{\prime} \rightarrow(K \rightarrow(A \rightarrow B))
$$

and so on ad infinitum. This problem, then, would seem to be resolved by 
assuming that every entailment is "absolute", i.e., it is "absolute" relative to some unstated but understood set of conditions such as an intuitive understanding of human language. A similar solution is to assume that every antecedent of an entailment contains these conditions implicitly.

\section{Other Applications}

D1 Chisholm on Doing Otherwise Chisholm [3] has asserted that the statement

1. He could have been in Boston now.

is not (essentially) equivalent to any statement of the form

2. He would have been in Boston now if he had undertaken (chosen, willed, tried, set out) to be in Boston now.

To explain, let us rewrite statements 1 and 2 as

1.1 He could have done $x$.

2.1 If $y$ occurred, he would have done $x$.

Chisholm's rationale may then be expressed essentially as follows: Suppose $y$ is impossible, but suppose he would have done $x$ iff $y$ occurred. Then he could not have done $x$, which is obviously inconsistent with saying he could have done $x$, although not inconsistent with saying that if $y$ occurred, then he would have done $x$. Hence, statements 1 and 2 are not equivalent.

It is easy to show that Chisholm's argument fails if we consider it from the standpoint of relative modality. In particular, suppose that $y$ is impossible with respect to $p$, i.e., that $p \rightarrow-y$. But since $x$ iff $y$, clearly $p \rightarrow-x$, i.e., $x$ is impossible with respect to $p$. But this does not entail that $x$ is impossible with respect to some other condition-say $q$; hence, to say, as Chisholm effectively does, that he could not (with respect to $p$ ) have done $x$ is not inconsistent with saying that he could have done $x$ with respect to $q$.

D2 Necessity and Contingency Many philosophers hold that every statement is either "contingent" or "necessary", where by the former is meant a statement which could have had a truth-value different from the one it actually has, and where by the latter is meant a statement which could not have had a truth-value different from what it has. Now if we hold, as Aristotle did, that every statement about the past is "necessary", and if we also hold that all statements, including ones about the future, are already true or false ${ }^{3}$ then, since all statements about the future were either true or false in the past, it follows that all statements whatsoever are "necessary" and none are "contingent". For example, if it were true in the past that there will be a sea-battle tomorrow, then it is a true (and hence a necessary) statement that there will be a sea-battle tomorrow; but this conflicts with what we would normally wish to say in the present about a future possibility, namely, that it could be otherwise, and thus that, in the present, it is contingent. 
Now much as in the problem of "he could have done otherwise" discussed in section D1, relative modality can offer us a credible way out of the above dilemma. To be specific, we may say that, relative to $a$ complete knowledge of the future (or relative to the set of all true statements about the future), any statement about the future is necessary (i.e., necessarily true or necessarily false); but relative to our knowledge of the future in the present, a statement about the future is contingent. This reasoning, however, suggests a more general conclusion, to wit: Every statement is both "necessary" and "contingent". That is, if $S$ is a statement, then any statement which implies $S$ is a statement with respect to which $S$ is necessary; while on the other hand, since every statement makes some true or false assertion about the way things are, (the truth or falsity of) $S$ is thus contingent on the way things are.

One important application of the above theory is in dealing with the problem, discussed by Prior [11], p. 10, that quantified modal logic seems to be a "pointless complication unless we can give meaning to such forms as 'For some individual $x$, it is necessary that $x$ should $\phi$ '." This is explained by saying that, while it is meaningful to speak of general terms as having necessary qualities (Men are necessarily rational as a result of the definition "man is a rational animal"), "no such sense could be given to the statement that this object before me, say John Jones, is essentially rational but only accidentally earth-dwelling ... we cannot say of any particular individual that it is necessary that he be either rational or anything else." Now our above theory is relevant to this problem in that it permits us to say that John Jones is essentially, or necessarily, rational relative to the statement that John Jones is a man and that men are, by definition, rational. (Similarly, of course, we can say that John Jones is accidentally, or contingently, earth-dwelling, since that is what happens to be the case.)

In conclusion, it seems appropriate to note that the law of contradiction is only relatively necessary, despite the "transcendental argument" that its denial presupposes it. ${ }^{4}$ This, of course, is because the law of contradiction is necessary relative to, among other things, the knowledge required to use or understand it.

D3 Deriving "Ought" from "Is" Ever since the time of David Hume, empirical-minded philosophers have been distinguished by (among other things) the habit of applying the sobriquet "naturalistic fallacy" to the belief that statements of what ought to be can be derived from statements of what $i s$. The reason such philosophers have been so disdainful of this belief is that scientific investigation of nature apparently yields only natural physical laws, and not moral ones; so from this point of view it seems that nature is essentially amoral, and thus that morality cannot be derived from it or justified on the basis of it. However, if one considers the fact that human societies do develop moral laws, and that the behavior of societieslike that of all other objects-is merely the result of natural law, then it seems that there may after all be a significant sense in which the "is" of nature yields the "ought" of morality. 
Now the best resolution to the above controversy, so it seems, is to observe that "ought" "statements can be translated into statements of relative necessity. (For example, "You ought to do good works" can be reasonably translated as "It is necessary relative to your getting to heaven (or being loved, or producing the greatest good for the greatest number) that you do good works.") Statements of relative necessity, however, depend for their truth purely on empirical or logical facts (such as the facts-if they are facts-that God rewards do-gooders, or that everyone's doing good implies that the greatest good for the greatest number will be accomplished). Accordingly, we see that statements of what "ought to be", by being statements of relative necessity, are nothing more than statements of what "is". The "naturalistic fallacy", therefore, does not appear to be quite so fallacious after all.

D4 Armchair Astronomy The following argument has caused considerable concern among philosophers:

1. $9=$ the number of planets

2. Necessarily, $9>7$

3. Therefore, necessarily the number of planets $>7$.

Now the cause for concern with the above argument is that, while statements 1 and 2 are held harmless, 3 is considered not only false, but also a demonstration of the number 9's "referential opacity", which is merely an opaque way of saying that all equivalents are equal, but some are more equal than others. Now what needs to be said about the above argument is first, that the necessity of $9>7$ is a necessity relative to mathematics, but that the necessity of statement 3 is ambiguous, i.e., it could be considered an empirical necessity (which good empiricists do not accept), in which case we would (as good empiricists) consider statement 3 to be false; or it could be considered a mathematical necessity, in which case 3 would be true; or it could be considered a necessity relative to the statements which truly describe the world, in which case it would also be true. But the above argument's most important lesson, perhaps, is that no equality is unconditional, i.e., that no things which are said to be "equal" are substitutable for one another in every context.

D15 Nested Modalities Such modal expressions as "necessarily necessary", "necessarily possible", and "possibly necessarily possible" are known as nested modalities. According to Prior [11], p. 8, there is, among philosophers, a "not uncommon view that nested modal expressions . . . are meaningless." Despite this view, however, such expressions appear frequently in ordinary language. For example, in a recent article, Charles Petit [9] stated that

1. ". . . stable nuclei with up to ten thousand protons and neutrons may be possible."

but this is merely another way of saying that 
2. It is possible that it will be possible that stable nuclei with ten thousand protons and neutrons will be (i.e., will exist).

But how are we to interpret such statements? Are we, for example, to represent statement 2 as

3. $(p P a) P a$

which of course is equivalent to

4. $-(a \rightarrow(a \rightarrow-p))$.

The answer would seem to be that statement 3 is not correct, for if we assume $^{7}$ that statements of the form $x \rightarrow(y \rightarrow z)$ are equivalent in ordinary language to statements of the form $(x, y) \rightarrow z$, then statement 4 becomes

5. $-(a \rightarrow-p)$

but this makes the modal nesting superfluous; whereas if we leave statement 4 undisturbed, we seem to condone self-reference, ${ }^{5}$ since this expression reads ". . . $a$ implies that $a$ implies that . . .". Another alternative, therefore, is to assume that statement 3 should be interpreted as

6. $(p P a) P b$.

This, it may be observed, seems a reasonable interpretation of statement 2, and thus of 1 , for 1 seems to be saying something on the order of

7. It is possible that adequate machinery will be developed to produce nuclei with ten thousand protons and neutrons; and if this machinery is in fact produced, then it will be possible (in a different sense of "possible") to produce such nuclei.

\section{NOTES}

1. von Wright [13] devotes a chapter to developing "A New System of Logic" which is similar in its fundamentals to what is developed here. The primary difference between his system and the present one is that von Wright permits "absolute" modality, which I do not; and this prevents him from being able to define modality-as it is done here-in terms of implication, or "entailment". (Actually, von Wright was interested in defining entailment in terms of modality-see pp. 175-176.) His system also leads to "implicational paradoxes" which are avoided in the system I have developed in [2].

2. Note that both trichotomies ought to be considered relativistic, the latter being taken as relative to certain information or knowledge.

3. The theory that all statements about the future are either true or false is known as the "full future theory"; whereas the theory that such statements have no truth value is known as the "empty future theory".

4. Note that while its denial may presuppose it, the failure to use it does not.

5. For an in-depth discussion of the undesirability of self-reference, see my [1], ch. 7. 
6. It may be noted that our efforts here are in accord with those of some philosophers who have treated modal expressions as metalogical, and in particular who have taken "necessarily $p$ " as the metalogical statement that $p$ is "provable from statement $s$ ". $C f$. [11], pp. 8-9.

7. I have referred to this assumption in [2], sec. H, as the First Conjunction Postulate.

\section{REFERENCES}

[1] Bryant, J., Systems Theory and Scientific Philosophy, Gordon and Breach, forthcoming.

[2] Bryant, J., "The truth-functional/containment theory of logical implication," unpublished doctoral project.

[3] Chisholm, R. M., "He could have done otherwise," in [5].

[4] Edwards, P., The Encyclopedia of Philosophy, Macmillan, 1967.

[5] Gill, J., Philosophy Today \#1, Macmillan, 1968.

[6] Hamlyn, D., "Contingent and necessary statements," in [4], vol. 2, pp. 198-205.

[7] Hume, D., An Enquiry Concerning Human Understanding, London, 1748.

[8] Lewis, C. I. and C. H. Langford, Symbolic Logic, New York, 1932.

[9] Petit, C., "Startling theory for an unknown matter," San Francisco Chronicle, Jan. 26, 1976.

[10] Prior, A. N., "Logic, deontic," in [4], vol. 4, pp. 509-513.

[11] Prior, A. N., "Logic, modal," in [4], vol. 5, pp. 5-12.

[12] Taylor, R., "Causation," in [4], vol. 2, pp. 56-66.

[13] von Wright, G. H., Logical Studies, Routledge, London, 1957.

Plymouth Meeting, Pennsylvania 